\title{
Cerebral amyloid angiopathy mimicking central nervous system metastases: a case report
}

\author{
Christopher DeZorzi ${ }^{*}$ Ruth Fernandez-Ruiz, Sarika Gupta and Katherine Harris
}

\begin{abstract}
Background: This case describes an unusual presentation of an intracranial hemorrhage first thought to be metastatic disease on computed tomography and magnetic resonance imaging. The healthcare team completed an exhaustive search for a primary malignancy that was negative. Final diagnosis on brain biopsy showed intercranial hemorrhage secondary to cerebral amyloid angiopathy. With an increasing number of elderly patients and the rising cost of health care, this case can serve as a reminder to clinicians about their own responsibilities in limiting the cost of health care.

Case presentation: This is a case report about a 72-year-old white woman with an intracranial hemorrhage secondary to cerebral amyloid angiopathy. The brain lesions on computed tomography/magnetic resonance imaging mimicked a metastatic process until a brain biopsy could give a definitive diagnosis that was completely unexpected. Cerebral amyloid angiopathy is a rare cause of intracerebral hemorrhage and this diagnosis is important to consider in older patients on anticoagulation.

Conclusions: Cerebral amyloid angiopathy is a rare diagnosis but should be considered in elderly patients on anticoagulation presenting with imaging findings consistent with intracerebral hemorrhage. While metastatic disease is a more common cause of intracerebral hemorrhage, cerebral amyloid angiopathy should remain in the differential diagnosis. This case report serves as a teaching point to clinicians in cases involving an older patient on anticoagulation.
\end{abstract}

Keywords: Clinical pathology, Cerebral amyloid angiopathy, Dementia, Intracranial hemorrhage, Alzheimer's disease

\section{Background}

Cerebral amyloid angiopathy (CAA) is characterized by amyloid deposition in cerebral blood vessels. This deposition consists of $\beta$-amyloid peptide in the media of small and medium-sized cerebral arteries. Marked deposition can lead to necrosis of the vessel walls and hemorrhage. It is commonly associated with Alzheimer's disease (AD) but most patients with hemorrhage related to CAA do not have $\mathrm{AD}$ [1]. It can occur as a sporadic disorder, sometimes in association with $\mathrm{AD}$, or as a familial syndrome. CAA is a cause of spontaneous cerebral hemorrhage especially in the elderly and may present as a sudden unexpected death in an older person. Patients on orally administered anticoagulation with CAA are also especially prone to intracranial

\footnotetext{
* Correspondence: christopher-dezorzi@uiowa.edu

University of lowa Hospitals and Clinics, 200 Hawkins Dr, lowa City, IA 52242, USA
}

(c) The Author(s). 2018 Open Access This article is distributed under the terms of the Creative Commons Attribution 4.0 International License (http://creativecommons.org/licenses/by/4.0/), which permits unrestricted use, distribution, and reproduction in any medium, provided you give appropriate credit to the original author(s) and the source, provide a link to the Creative Commons license, and indicate if changes were made. The Creative Commons Public Domain Dedication waiver (http://creativecommons.org/publicdomain/zero/1.0/) applies to the data made available in this article, unless otherwise stated. orally administered anticoagulation would be at high risk of bleeding if they had CAA. The definitive diagnosis of this condition still requires pathological examination of brain tissue. The prevalence of CAA is markedly age dependent. It can be identified pathologically in up to $35 \%$ of brains from individuals aged 85 years or older. One-third of these older patients with CAA are affected to the extent associated with hemorrhage [2, 3]. In younger patients, the prevalence of moderate to severe CAA is $2.3 \%$ for patients between the ages of 65 and 74 and $8.0 \%$ between the ages of 75 and 84 [3]. Although hypertensive vasculopathy is the leading cause of $\mathrm{ICH}$ in the deep hemispheric regions of the brain, CAA may be more common for lobar ICH [4]. This is a cause for concern, considering the lack of treatment options we have for CAA compared with hypertensive vasculopathy. 


\section{Case presentation}

A 72-year-old white woman with a medical history of recurrent pneumonia, pulmonary embolism 1 month ago (currently on warfarin therapy), and coronary artery disease presented from an independent living facility with altered mentation. She had no known family history of CAA or $\mathrm{ICH}$ and had not been brought into the Emergency Room (ER) for confusion previously. At baseline, she was very independent, living on her own, and was able to perform all her activities of daily living (ADL). Her family indicated that she started to have worsening altered mental status, to the point of being found profoundly confused by staff in the lobby of her living facility, prompting her urgent transfer to a local hospital by the Emergency Medical Service. On presentation, she was febrile to $39.9{ }^{\circ} \mathrm{C}$ and had right-sided deficits with repetitive speech. A computed tomography (CT) scan (Fig. 1) was concerning for multiple areas of hemorrhage, therefore, she was transferred to our tertiary hospital. She arrived hemodynamically stable with no focal deficits on neurologic examination. Magnetic resonance imaging (MRI) of her brain showed multiple hemorrhagic ring-enhancing lesions with surrounding vasogenic edema, highly suspicious for hemorrhagic metastasis (Figs. 2 and 3). In the Medical Intensive Care Unit, she received 4-factor prothrombin complex concentrate and vitamin $\mathrm{K}$ to reverse her international normalized ratio (INR), broad-spectrum antibiotics for the possibility of the lesions being infectious, intravenously administered dexamethasone to reduce brain edema, and levetiracetam after an electroencephalogram (EEG) showed a seizure tendency. Over the next few days she had no signs or obvious source of infection, so an exhaustive workup in an attempt to find a primary

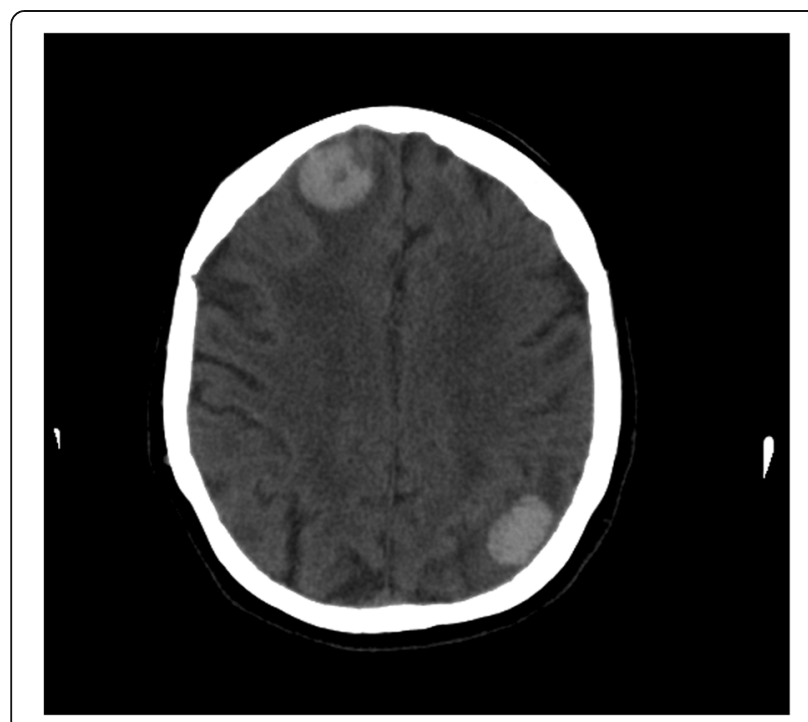

Fig. 1 Computed tomography scan of the brain showed multiple areas of hemorrhage

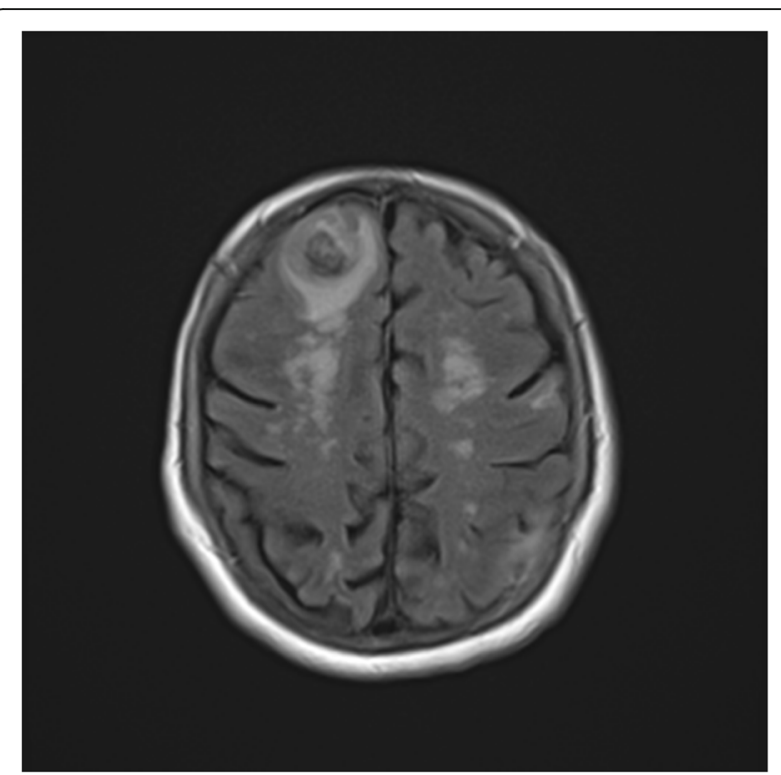

Fig. 2 Magnetic resonance imaging of the brain was remarkable for multiple hemorrhagic ring-enhancing lesions with surrounding vasogenic edema, highly suspicious for hemorrhagic metastases versus abscesses

malignancy was initiated. A CT of her chest, abdomen, and pelvis resulted in no findings suggestive of a primary lesion. A thyroid ultrasound, mammogram, and positron emission tomography (PET)/CT (skull base to upper thigh) were also negative (Fig. 4). Further evaluation with dermatology showed basal cell carcinoma of the nasal wall, but no evidence of melanoma. Finally, the neurosurgery team was consulted to discuss options for tissue diagnosis and a brain

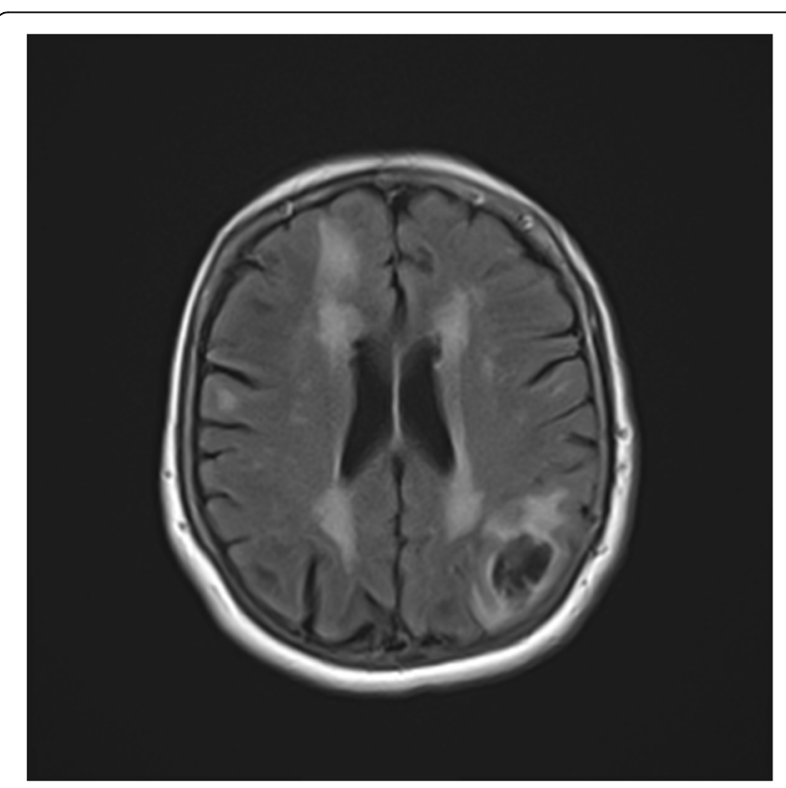

Fig. 3 Magnetic resonance imaging of the brain was remarkable for multiple hemorrhagic ring-enhancing lesions with surrounding vasogenic edema, highly suspicious for hemorrhagic metastases versus abscesses 


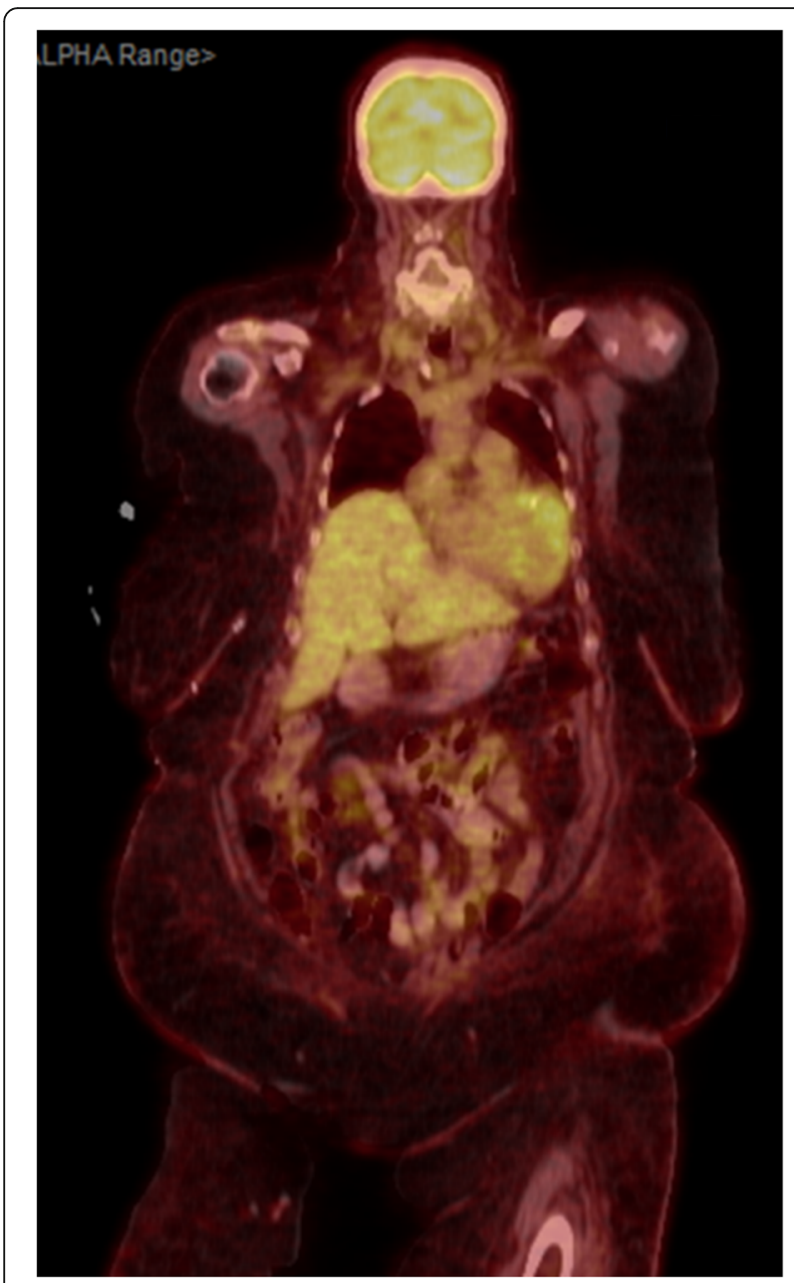

Fig. 4 Positron emission tomography scan was negative as a part of workup to look for a primary lesion

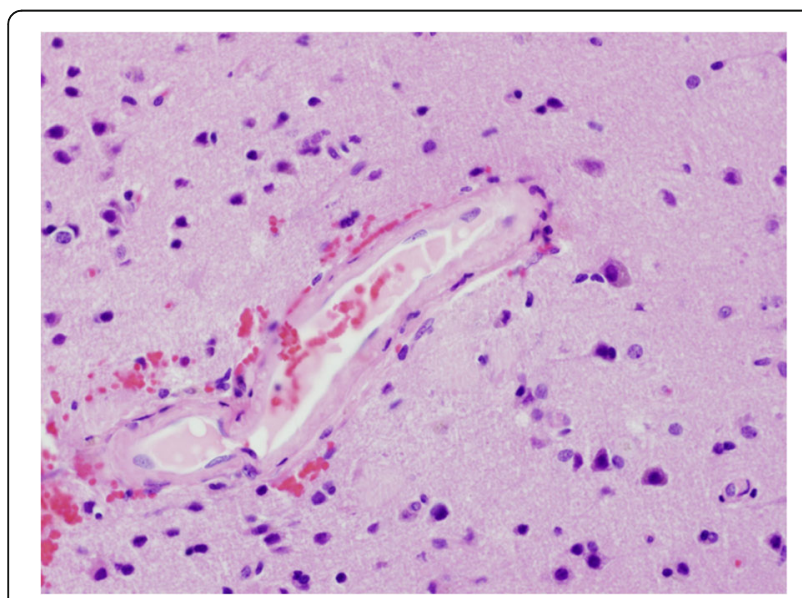

Fig. 5 Hematoxylin and eosin stained section $(\times 40)$ illustrating hyalinization of intra-parenchymal arterioles

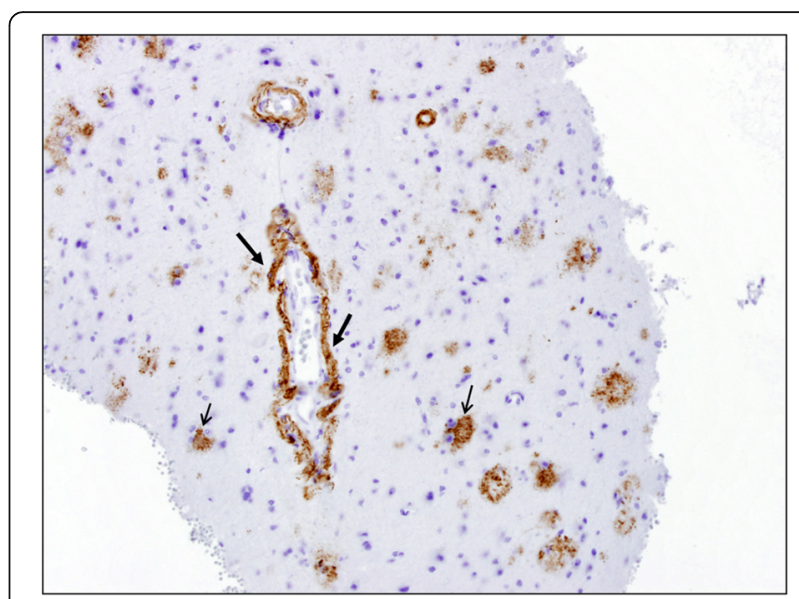

Fig. 6 Immunohistochemical staining $(\times 20)$ highlights the amyloid beta deposition in vessel walls (thick arrows). Scattered amyloid plaques are also present (thin arrows)

tissue biopsy was obtained. The results showed amyloid angiopathy and an organized hematoma with gliosis but no signs of neoplasm (Figs. 5 and 6). Discussions among the primary care team and specialty teams then changed focus to anticoagulation due to her recent pulmonary embolism. Given her new diagnosis, she would be a very high-risk anticoagulation candidate due to her increased risk of intracerebral hemorrhage, so an inferior vena cava filter was placed. For discharge, she was prescribed a dexamethasone taper, continued on levetiracetam, scheduled for a follow-up MRI in 1 month, and was discharged to a skilled nursing facility with slightly increased confusion from her baseline but much improved from her presentation. On follow-up 1 year later she was showing increasing signs of dementia. She lives in an assisted living facility but maintains a majority of her ADL independently.

\section{Discussion}

As the medical field has developed more advances and pharmaceutical options for hypertensive control, the expected decrease in the incidence of $\mathrm{ICH}$ has not followed. The overall incidence is actually stable, which could be secondary to orally administered anticoagulationassociated bleeds in our ageing population [5]. Warfarin is the most commonly used orally administered anticoagulant and works by inhibiting vitamin K-dependent coagulation factors II, VII, IX, and X. The anti-thromboembolic benefits of anticoagulation have been proven significantly useful in several cardiac-related conditions and for venous thromboembolisms even beyond its risk of bleeding [6]. The exact nature of orally administered anticoagulantassociated ICH is likely intertwined with the effects of ageing, genetics, and other concurrent vascular conditions on the brain. Older patients who have CAA in addition to anticoagulation will be especially prone to $\mathrm{ICH}$. 
Of interest, in this clinical scenario CT and MRI actually led the primary team toward chasing after the wrong underlying etiology of the ICH. The reports from radiology for both the $\mathrm{CT}$ and MRI stated that the lesions were most consistent with metastasis. After the primary team reviewed this with radiology, a complete and very expensive workup to find the primary lesion followed. This exhaustive workup creates a huge burden and contributes to the rising cost of health care. Although in this scenario it was appropriate to attempt to find primary malignant lesions, this case can be used as a reminder to keep in mind the possibility of ICH due to CAA especially in the presence of an older patient on anticoagulation. It is important to remember ICH is not always associated with hypertension or Alzheimer's dementia.

Another topic in this clinical scenario is whether to restart the orally administered anticoagulation. There is an elevated risk of CAA and ICH in the elderly, which is a given reason to hold anticoagulation. This is counterbalanced by a parallel increased risk of thromboembolic stroke with increasing age [2]. The benefits of stroke prevention, or prevention of pulmonary embolism in this case, many times outweigh the risks of bleeding in patients when deciding to start or continue anticoagulation. In this case, it was decided that anticoagulation risks posed a larger problem than our patient's previous pulmonary embolism. Given the large role of CAA as an underlying cause of anticoagulant-related $\mathrm{ICH}$ [7] and the high rate of $\mathrm{ICH}$ recurrence in patients with CAA [8], it is recommended that long-term anticoagulation not be prescribed for survivors of suspected CAA-related ICH who have non-valvular atrial fibrillation $[5,9]$. It is unknown, but likely, that the same risks would outweigh the benefits in a patient with a history of pulmonary embolism.

\section{Conclusions}

- CAA is a rare cause of intracerebral hemorrhage

- This diagnosis is important to consider in older patients on anticoagulation presenting with $\mathrm{ICH}$

- A lack of hypertension can be a clue to CAA causing ICH

- The use of anticoagulants increases the risk of brain hemorrhage in patients with CAA

- With the knowledge of this case report, clinicians should now be able to devise broader differential diagnoses in similar situations

Acknowledgements

Not applicable.

Funding

There is no source of funding in preparing this case report.
Availability of data and materials

All data generated or analyzed during this study are included in this published article.

\section{Authors' contributions}

$\mathrm{CD}$ wrote and did research for the manuscript. RF helped edit the manuscript. SG prepared the pathology-related excerpts of the manuscript and figures. $\mathrm{KH}$ helped edit the manuscript. All authors read and approved the final manuscript.

Ethics approval and consent to participate

Not applicable.

\section{Consent for publication}

Written informed consent was obtained from the family of the patient (due to increasing dementia in the patient herself) for publication of this case report and any accompanying images. A copy of the written consent is available for review by the Editor-in-Chief of this journal.

\section{Competing interests}

The authors declare that they have no competing interests.

\section{Publisher's Note}

Springer Nature remains neutral with regard to jurisdictional claims in published maps and institutional affiliations.

Received: 21 July 2017 Accepted: 18 March 2018

Published online: 14 May 2018

\section{References}

1. Greenberg SM, Edgar MA. Case Records of the Massachusetts General Hospital (Case 22-1996). N Engl J Med. 1996;335:189-96.

2. Rosand J, Hylek EM, O'Donnell HC, Greenberg SM. Warfarin-associated Hemorrhage and Cerebral Amyloid Angiopathy: A Genetic and Pathologic Study. Neurology. 2000;55(7):947-51. Web

3. Greenberg SM, J-PG V. Diagnosis of cerebral amyloid angiopathy. Sensitivity and specificity of cortical biopsy. Stroke. 1997;28:1418-22

4. Cervera A, Amaro S, Chamorro A. Oral Anticoagulant-associated Intracerebral Hemorrhage. J Neurol. 2012;259(2):212-24.

5. Gurol ME, Greenberg SM. Management of Intracerebral Hemorrhage. Curr Atheroscler Rep. 2008;10(4):324-31. Web

6. Albers GW, Amarenco P, Easton JD, Sacco RL, Teal P. Antithrombotic and thrombolytic therapy for ischemic stroke: American College of Chest Physicians evidence-based clinical practice guidelines (8th Edition). Chest. 2008;133(6 Suppl):630S-69S.

7. Rosand J, Hylek EM, O'Donnell HC, Greenberg SM. Warfarin-associated hemorrhage and cerebral amyloid angiopathy: a genetic and pathologic study. Neurology. 2000:55:947-51.

8. Greenberg SM, Eng JA, Ning M, et al. Hemorrhage burden predicts recurrent intracerebral hemorrhage after lobar hemorrhage. Stroke. 2004;35:1415-20.

9. Eckman $\mathrm{MH}$, Rosand J, Knudsen KA, et al. Can patients be anticoagulated after intracerebral hemorrhage? A decision analysis. Stroke. 2003;34:1710-6.

Submit your next manuscript to BioMed Central and we will help you at every step:

- We accept pre-submission inquiries

- Our selector tool helps you to find the most relevant journal

- We provide round the clock customer support

- Convenient online submission

- Thorough peer review

- Inclusion in PubMed and all major indexing services

- Maximum visibility for your research 